Original Research Article

\title{
Comparative evaluation of levetiracetam and valproic acid as monotherapy on cognitive impairment in patients of epilepsy
}

\author{
Shreshth Khanna ${ }^{1}$, Suman Bala ${ }^{1 *}$, Yashpal Singh ${ }^{2}$, Taruna Sharma ${ }^{1}$, Juhi Kalra ${ }^{1}$, \\ D. C. Dhasmana ${ }^{1}$, Saurabh Kohli ${ }^{1}$
}

${ }^{1}$ Department of Pharmacology, Swami Rama Himalayan

University, Dehradun,

Uttarakhand, India

${ }^{2}$ Department of Neurology,

Swami Rama Himalayan

University, Dehradun,

Uttarakhand, India

Received: 04 January 2019

Accepted: 08 March 2019

*Correspondence to:

Dr. Suman Bala,

Email:

Yashsuman2000@gmail.com

Copyright: (C) the author(s), publisher and licensee Medip Academy. This is an openaccess article distributed under the terms of the Creative Commons Attribution NonCommercial License, which permits unrestricted noncommercial use, distribution, and reproduction in any medium, provided the original work is properly cited.

\begin{abstract}
Background: Cognitive decline with AEDs (Anti-epileptic drugs) is associated with learning and memory deficits especially in the younger age group. The data regarding the impact of levetiracetam and valproic acid as monotherapy on cognition in epileptic patients is scarce. The present study was done for evaluation of cognitive decline associated with the use of AEDs.

Methods: Present study was a prospective study on 60 patients on AEDs for a period of 12 weeks. Patients were enrolled from the Department of Neurology, Swami Rama Himalayan University, Dehradun, Uttarakhand, India and divided into group A (levetiracetam) and group B (valproic acid) with 30 patients in each group. Permission from the institutional ethics committee and written informed consent was taken from all the patients. They were analyzed for cognitive impairment using MMSE and MoCA scales at baseline and 12 weeks.

Results: The mean duration of disease was $2.13 \pm 1.1$ years and $2.08 \pm 1.1$ years and mean age of the patients was $14.67 \pm 1.9$ years in group A and 16.20 \pm 1.6 years in group B. GTCS was present in 31 patients $(52 \%)$ followed by partial seizures in 29 patients (48\%). The mean change in the MMSE scores from baseline to 12 weeks was significant in group A $1.30 \pm 1.1$ ( $p$ value $<0.05$ ) and change group B was $-0.20 \pm 1.4$ not statistically significant. The mean change was observed in MoCA scores from baseline to 12 weeks was significant in both groups A and B by $1.17 \pm 1.1$ and $-0.70 \pm 1.1$ respectively ( $P$ value $<0.05$ ).

Conclusions: Patients on levetiracetam showed cognitive improvement, whereas patients on valproic acid showed a decline in the MMSE and MoCA scores.
\end{abstract}

Keywords: Cognitive impairment, Epilepsy, MMSE, MoCA

\section{INTRODUCTION}

Epilepsy is one of the most common causes of neurological diseases. According to WHO 50 million people are suffering from epilepsy. ${ }^{1}$ Patients of epilepsy usually undergo long-term treatment for years with antiepileptic drugs (AEDs). ${ }^{2}$ Anti-epileptic drugs (AEDs) are known to impact the cognitive abilities in the patient of epilepsy. The major cognitive adverse effects associated with AEDs are impaired attention, vigilance, slowing of mental and psychomotor speed. Cognitive impairment as an entity itself puts a depressing impact on the self-confidence of the patient. ${ }^{3}$ Long-term consequences of AED in term of cognitive impairment are obvious in the early ages of life. ${ }^{4}$ Despite the large number of newer agents, most neuropsychological studies have compared newer drugs to older AEDs with high risk cognitive impairment or against newer drugs at dosages that do not reflect current prescribing pattern making, the comparative effect of newer agents unclear. ${ }^{5}$ Comparative Data are lacking on 
their effects on cognition, hence this study is planned for a better understanding of comparative evaluation of levetiracetam and valproic acid as mono-therapy on cognitive impairment in patients of epilepsy.

\section{METHODS}

Present study was observational and follows up study with two parallel groups conducted in newly diagnosed patients of epilepsy as per the ILAE classification. ${ }^{6} \mathrm{~A}$ minimum of 60 patients were required with 30 patients in each group based on a previous study. The study was approved by the institutional ethical committee.

\section{Participants}

Eligible participants were male and females in the age group of 12-18 years newly diagnosed with epilepsy as per the ILAE classification. The patients were recruited from the outpatient unit of the Department of Neurology, Swami Rama Himalayan University, Dehradun, Uttarakhand, India, after obtaining a prior written informed consent. Follow up time for the patients were 12 weeks. Patients with any progressive CNS disease, serious cardiac abnormality, underlying malignancy, hypersensitivity to any of the study drugs, uncontrolled severe concurrent illness, abnormal liver and kidney functions, pregnant and lactating females, history of seizures due to drugs, alcohol, acute medical illness, psychiatric disorders and patients leaving the study due to any reasons will be excluded from final analysis. Demographic profile and detailed history were obtained from each recruited patient, this included family history, educational status, age of onset of epilepsy, duration of disease, personal habits. A general physical examination was performed, and blood pressure was recorded EEG and $\mathrm{CT}$ heads was done. Blood test (haematological and biochemistry were done before starting of the treatment. Study subjects included in the study were divided into two groups of 30 each. The drugs were given to subjects on the basis of physician's discretion.

The dose ranges of the two drugs at the start of the study were as follows for levetiracetam (LEV) 500-2000 mg/day and for valproic acid (VPA) 300-1000 mg/day. After recruitment patients were assessed for the cognitive impairment based on the MMSE and MoCA questionnaires. Patients were evaluated at 0,6 and 12 weeks or earlier as the need arose. For efficacy and safety, they were assessed on each visit with the help of patientmaintained seizure diary and self-reporting of adverse drug reaction.

\section{Study measurements}

Patient reported outcomes were assessed by well validated measures. The MMSE and MoCA questionnaires were used to describe cognitive impairment in patients. The patients were asked to fill both the questionnaire scales. Scores for MMSE and MoCA scales range from a minimum of 0 to a maximum of 30 . Lower the score worse is the cognitive outcome. A cut-off score of less than 24 on the MMSE scale and less than 26 on the MoCA scale was used as an indicator of cognitive impairment. These cut off scores were chosen based on a previous study assessing cognitive performance. ${ }^{7}$

Data regarding these outcomes were collected at baseline and 12 weeks to assess cognitive impairment with the use of anti-epileptic drugs (AEDs).

\section{End points}

\section{Primary parameters}

- $\quad$ Change in the MMSE score,

- Change in montreal cognitive assessment scale (MoCA).

\section{Secondary parameters}

- Freedom from seizure: this will refer to number of seizure episodes till the last follow up using seizure diary,

- Adverse drug reaction as per check list along with spontaneous (ADRs) will be recorded.

In assessment of safety, a checklist of adverse drug reactions was prepared according to the most common adverse events due to study drugs. Adverse drug reactions were recorded at every visit of the patient to the OPD. Seizure diary was used by the patient to self-record his/her experiences every week and to observe the improvement or deterioration in frequency of seizures, duration of episodes, duration of post-ictal confusion and any seizure related injury.

\section{Data management}

Data management and analysis was done using Microsoft Excel 2007 and IBM SPSS version 20.0. Demographic data was presented as either frequency or Mean \pm SD. Intragroup comparison was done using paired sample student ttest and inter-group analysis was done using unpaired student t-test. Adverse events were interpreted and analyzed using descriptive statistics and chi-square test.

\section{RESULTS}

\section{Patient characteristics}

From January 2017 to December 2017, 120 patients were screened from which sixty patients who fulfilled the eligibility criteria (42 male, 18 female) were enrolled in this study (Table 1). The patients were newly diagnosed epileptic patients on monotherapy on either valproic acid or levetiracetam based on physician's discretion. Patients were followed up for a period of 12 weeks after enrolment for cognitive impairment due to AEDs. 
Table 1: Demographic profile and baseline characteristics of patients of epilepsy in both the groups.

\begin{tabular}{|c|c|c|c|c|}
\hline \multicolumn{3}{|c|}{ Demographic profile } & Group A (LEV)(n=30) & Group B (VPA) $(\mathrm{n}=\mathbf{3 0})$ \\
\hline \multicolumn{3}{|c|}{ Total no. of patients } & 30 & 30 \\
\hline \multicolumn{3}{|c|}{ Age in years $($ mean $\pm S D)$} & $14.67 \pm 1.882$ & $16.20 \pm 1.648$ \\
\hline \multicolumn{3}{|c|}{ Sex (male/female) } & $18 / 12$ & $24 / 6$ \\
\hline \multicolumn{3}{|c|}{ Smoker/non-smoker } & $4 / 26$ & $7 / 23$ \\
\hline \multicolumn{3}{|c|}{ Alcoholic/non-alcoholic } & $8 / 22$ & $5 / 25$ \\
\hline \multicolumn{3}{|c|}{ Residence (urban/rural) } & $13 / 17$ & $20 / 10$ \\
\hline \multicolumn{3}{|c|}{ Religion (Hindu/Muslim/Christian/ Sikh) } & $25 / 4 / 1 / 0$ & $26 / 3 / 0 / 1$ \\
\hline \multicolumn{3}{|c|}{ Education (above 10/less than 10) } & $6 / 24$ & $12 / 18$ \\
\hline \multirow{4}{*}{ Parameters } & \multirow{2}{*}{ Type of seizures } & GTCS & 6 & 25 \\
\hline & & Partial & 24 & 5 \\
\hline & \multirow{2}{*}{ Family history } & Present & 5 & 7 \\
\hline & & Absent & 25 & 23 \\
\hline \multicolumn{3}{|c|}{ Duration of disease (years) } & $2.13 \pm 1.080$ & $2.08 \pm 1.089$ \\
\hline \multicolumn{3}{|c|}{ Frequency of seizures } & $1.93 \pm 0.907$ & $2.63 \pm 1.326$ \\
\hline
\end{tabular}

Table 2: Comparison of the change from baseline to 12 weeks in the mean MMSE score in group A (LEV) and group B (VPA).

\begin{tabular}{|lllllll|}
\hline \multirow{2}{*}{ MMSE parameters } & \multicolumn{2}{l}{ Group A $(\mathbf{L E V})(\mathbf{n}=\mathbf{3 0})$} & & \multicolumn{2}{l|}{ Group B $(\mathrm{VPA})(\mathbf{n}=\mathbf{3 0})$} \\
& Baseline & $\mathbf{1 2}$ weeks & Mean change & Baseline & 12 weeks & Mean change \\
\hline Orientation & $9.37 \pm 0.72$ & $9.87 \pm 0.35^{*}$ & $0.50 \pm 0.57^{*}$ & $9.40 \pm 0.77$ & $9.43 \pm 0.82$ & $0.03 \pm 0.67 \#$ \\
\hline Registration & $2.93 \pm 0.25$ & $2.93 \pm 0.25$ & \pm 0.00 & $2.87 \pm 0.35$ & $2.90 \pm 0.35$ & $0.03 \pm 0.32$ \\
\hline Attention and calculation & $4.10 \pm 0.71$ & $4.47 \pm 0.68^{*}$ & $0.37 \pm 0.56^{*}$ & $4.23 \pm 0.68$ & $4.17 \pm 0.79$ & $-0.07 \pm 0.64 \#$ \\
\hline Recall & $2.40 \pm 0.68$ & $2.47 \pm 0.63$ & $0.07 \pm 0.52$ & $2.63 \pm 0.49$ & $2.57 \pm 0.57$ & $-0.07 \pm 0.58$ \\
\hline Language & $7.07 \pm 0.74$ & $7.57 \pm 0.57^{*}$ & $0.50 \pm 0.73^{*}$ & $7.17 \pm 0.65$ & $7.03 \pm 0.76$ & $-0.17 \pm 0.53 \#$ \\
\hline Total MMSE & $25.80 \pm 1.5$ & $27.10 \pm 1.3^{*} \#$ & $1.30 \pm 1.02^{*}$ & $26.33 \pm 1.71$ & $26.10 \pm 1.8$ & $-0.20 \pm 1.40 \#$ \\
\hline
\end{tabular}

Values expressed as mean $( \pm \mathrm{SD}), *$ Student $\mathrm{t}$-test $\mathrm{p}<0.05$ was considered statistically significant intra-group, \#Student $\mathrm{t}$ - test $\mathrm{p}<0.05$ was considered statistically significant inter-group.

Table 3: Comparison of change in mean MOCA scores in group A (LEV) and group B (VPA) from baseline to 12 weeks.

\begin{tabular}{|lllllll|}
\hline $\begin{array}{l}\text { MOCA } \\
\text { parameters }\end{array}$ & Group A $(\mathrm{LEV})(\mathbf{n}=\mathbf{3 0})$ & \multicolumn{4}{ll}{ Group B $(\mathrm{VPA})(\mathbf{n}=\mathbf{3 0})$} \\
\hline Visuo-spatial & $4.50 \pm 0.572$ & $4.47 \pm 0.776$ & $-0.03 \pm 0.809$ & $4.57 \pm 0.626$ & $4.37 \pm 0.718$ & $-0.13 \pm 0.629$ \\
\hline Naming & $2.87 \pm 0.346$ & $2.97 \pm 0.183$ & $0.10 \pm 0.403$ & $2.90 \pm 0.305$ & $2.90 \pm 0.305$ & $-0.07 \pm 0.365$ \\
\hline Attention & $5.00 \pm 0.910$ & $5.60 \pm 0.675^{*}$ & $0.60 \pm 0.894^{*}$ & $5.43 \pm 0.935$ & $5.33 \pm 0.758$ & $-0.07 \pm 1.015$ \\
\hline Language & $2.10 \pm 0.305$ & $2.13 \pm 0.346$ & $0.03 \pm 0.414$ & $2.30 \pm 0.466$ & $2.37 \pm 0.490$ & $0.10 \pm 0.548$ \\
\hline Abstraction & $0.93 \pm 0.365$ & $1.20 \pm 0.407^{*}$ & $0.27 \pm 0.450^{*}$ & $1.27 \pm 0.450$ & $1.10 \pm 0.305$ & $-0.20 \pm 0.484$ \\
\hline Delayed recall & $3.37 \pm 0.765$ & $3.43 \pm 0.504$ & $0.07 \pm 0.907$ & $3.57 \pm 0.728$ & $3.23 \pm 0.430^{*}$ & $-0.27 \pm 0.691$ \\
\hline Orientation & $5.87 \pm 0.346$ & $5.93 \pm 0.254$ & $0.07 \pm 0.365$ & $5.83 \pm 0.379$ & $5.83 \pm 0.461$ & $-0.03 \pm 0.183$ \\
\hline Total MOCA & $24.93 \pm 1.112$ & $26.10 \pm 1.296^{*}$ & $1.17 \pm 1.147 *$ & $26.23 \pm 1.591$ & $25.53 \pm 1.655^{*}$ & $-0.70 \pm 1.119$ \\
\hline
\end{tabular}

Values expressed as mean $( \pm \mathrm{SD})$, Student t-test $* \mathrm{p}<0.05$ significant.

No statistically significant differences in between the groups were observed based on the basis of their baseline characteristics. The two groups differed only on the base of their personal history (alcoholism and smoking) (Table 1).

Significant improvements in MMSE sub scores were seen in all the parameters in the LEV group from baseline to 12 weeks except registration and recall parameter.
In the VPA group, improvement in the orientation and registration parameters was seen which were not statistically significant, the total score deteriorated from $26.33 \pm 1.709$ at baseline to $26.10 \pm 1.77$ at the end of 12 weeks but the change was not statistically significant (Table 2). Intra group comparison in groups A and B for total MOCA scores showed difference that was statistically significant from baseline to the end of the study at 12 
weeks. Significant Improvements in the scores were seen in attention and abstraction parameters in the LEV group ( $p$ <0.05). In the VPA group, all the parameters showed deterioration in the scores except the language parameter which showed improvement, but the change was not significant and only significant difference in the scores was seen in the delayed recall parameter $(\mathrm{p}<0.05)$. Total score significant improvement was seen from $24.93 \pm 1.112$ to $26.10 \pm 1.296$ in the LEV group ( $p<0.05)$, in the VPA group the total score deteriorated from $26.23 \pm 1.591$ to
$25.53 \pm 1.655$ which was also statistically significant $(\mathrm{p}<0.05)$ (Table 3).

On comparing MMSE score changes in both the groups in patients with partial seizures, there was improvement in the MMSE scores in patients of group A (LEV) when compared to MMSE scores in patients of group B (VPA) in all the parameters except recall which showed improvement, but which was not statistically significant (Table 3).

Table 4: Comparison of mean change in MMSE and MOCA scores between group A (LEV) and group B (VPA) based on the seizure type from baseline to 12 weeks.

\begin{tabular}{|lllll|}
\hline Parameters & Partial & & GTCS & Group B (VPA) \\
\hline MMSE parameters & Group A (LEV) & Group B (VPA) & Group A (LEV) & $0.04 \pm 0.735$ \\
\hline Orientation & $0.54 \pm 0.58$ & $0.00 \pm 0.00$ & $0.33 \pm 0.516$ & $0.04 \pm 0.200$ \\
\hline Registration & $0.00 \pm 0.00$ & $0.00 \pm 0.707$ & $0.00 \pm 0.00$ & $-0.12 \pm 0.66$ \\
\hline Attention and calculation & $0.38 \pm 0.576$ & $0.20 \pm 0.447$ & $0.33 \pm 0.516$ & $-0.16 \pm 0.554$ \\
\hline Recall & $0.17 \pm 0.381$ & $0.40 \pm 0.548$ & $-0.33 \pm 0.816^{*}$ & $-0.20 \pm 0.557$ \\
\hline Language & $0.38 \pm 0.647$ & $0.00 \pm 0.00$ & $1.00 \pm 0.894$ & $-0.32 \pm 1.314$ \\
\hline Total MMSE & $1.42 \pm 1.060$ & $0.40 \pm 1.817$ & $0.83 \pm 0.753$ & Group B (VPA) \\
\hline MOCA parameters & Group A (LEV) & Group B (VPA) & Group A (LEV) & $-0.16 \pm 0.688$ \\
\hline Visuo-spatial & $-0.08 \pm 0.830$ & $0.00 \pm 0.00$ & $0.17 \pm 0.753$ & $-0.04 \pm 0.351$ \\
\hline Naming & $0.08 \pm 0.408$ & $-0.20 \pm 0.447$ & $0.17 \pm 0.408$ & $-0.08 \pm 1.115$ \\
\hline Attention & $0.63 \pm 0.928$ & $0.00 \pm 0.00$ & $0.50 \pm 0.837$ & $0.08 \pm 0.572$ \\
\hline Language & $-0.04 \pm 0.359$ & $0.20 \pm 0.447$ & $0.33 \pm 0.516$ & $-0.20 \pm 0.500$ \\
\hline Abstraction & $0.29 \pm 0.464$ & $-0.20 \pm 0.447$ & $0.17 \pm 0.408$ & $-0.24 \pm 0.592$ \\
\hline Delayed recall & $0.21 \pm 0.884$ & $-0.40 \pm 1.140$ & $-0.50 \pm 0.837$ & $-0.04 \pm 0.200$ \\
\hline Orientation & $0.08 \pm 0.282$ & $0.00 \pm 0.00$ & $0.00 \pm 0.632$ & $-0.80 \pm 1.080$ \\
\hline Total & $1.13 \pm 1.11$ & $-0.20 \pm 1.304$ & $1.33 \pm 1.366$ & \\
\hline Values & Stin & & \\
\hline
\end{tabular}

Values expressed as mean $( \pm \mathrm{SD})$, Student t-test $* \mathrm{p}<0.05$ significant.

Table 5: Adverse events during the study period in Group A (LEV) and Group B (VPA).

\begin{tabular}{|lllll|}
\hline Adverse Effects & Group A $(\mathrm{LEV})(\mathbf{n}=\mathbf{3 0})$ & Percentage $(\%)$ & Group B (VPA) $(\mathbf{n = 3 0})$ & Percentage $(\%)$ \\
\hline Anorexia & - & - & 1 & 3 \\
\hline Decreased sleep & - & - & 1 & 3 \\
\hline Hair fall & - & - & 3 & 10 \\
\hline Headache & 2 & 7 & 7 & 23 \\
\hline Increased sleep & - & - & 5 & 17 \\
\hline Irritability & 4 & 13 & 2 & 3 \\
\hline Tiredness & 1 & 3 & 1 & 7 \\
\hline Weight gain & - & - & $22 *$ & 73 \\
\hline Total & 7 & 23 & 22 & 3 \\
\hline
\end{tabular}

Adverse events presented as frequency, $* \mathrm{p}<0.05$ statistically significant (chi-square).

On comparing MMSE score changes in both the groups in patients with GTCS, there was deterioration in the MMSE scores in patients of group B (VPA) when compared to MMSE scores in patients of group A (LEV) except in the registration parameter which showed improvement, but which was not statistically significant. On comparing MOCA score, changes in both the groups in patients of partial seizures, there was deterioration in the scores in patients of group B (VPA) when compared to MOCA scores in patients of group A (LEV) except visuospatial and language parameters which showed improvements in scores, but which were not statistically significant ( $p<0.05)$. On comparing MOCA score, changes in both the groups in patients with GTCS, there was deterioration in 
all the scores from baseline to 12 weeks in patients of group $\mathrm{B}$ (VPA) when compared to MOCA scores in patients of group A (LEV) (Table 4). Adverse events in group A (LEV) and group B (VPA) were well recorded. Adverse events recorded were 7 and 22 in group $A$ and $B$ respectively. This was found to be statistically significant $(p=0.00$, chi square $=15.017)$.

In the group A (LEV), adverse effects were noted in $23 \%$ of the patients. Most common adverse effects in the group A (LEV) was irritability (13\%) followed by headache $(7 \%)$ and tiredness $(3 \%)$.

In the group B (VPA), adverse effects were noted in $73 \%$ of the patients. Most common adverse effects in the group B (VPA) was headache (23\%) followed by somnolence $(17 \%)$, hair fall $(10 \%)$, irritability $(7 \%)$, anorexia $(3 \%)$, decreased sleep (3\%) and tiredness (3\%) (Table 5).

Seizure freedom was seen at 6 and 12 weeks in both the groups. It was seen that patients achieved a better seizure control in the levetiracetam group than valproic acid group at 6 weeks (Figure 1).

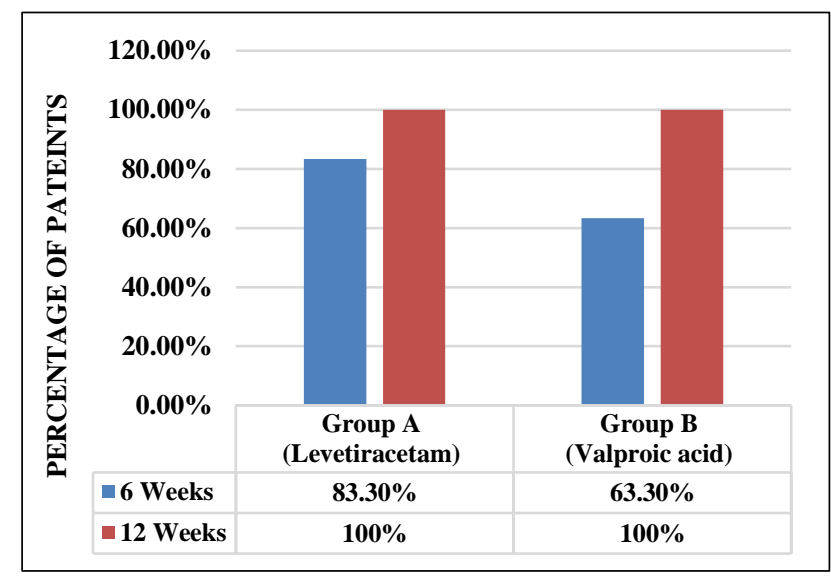

\section{Figure 1: Seizure freedom at 6 and 12 weeks in group A (LEV) and group B (VPA).}

\section{DISCUSSION}

The purpose of treatment of patients with epilepsy is freedom from seizures, normalizing his/her daily activities which are otherwise hindered and reducing the anticipation anxiety due to seizures due to epilepsy. Cognitive impairment due to disease as well as the AEDs used in the treatment add to the already existing problems in patients of epilepsy, reducing the patient's self-confidence and may impair the day to day activities.

General baseline demographic characteristics included in this study were age, gender and place of residence. Mean age of the study population in the present study was $14.67 \pm 1.882$ in the levetiracetam group and $16.20 \pm 1.648$ in the valproic acid group (Table 1) which was similar to the mean age (15.4 years) observed in a study on cognitive impairment by Sulheim D et al. ${ }^{8}$ The adolescent and young adult population forms a vulnerable group where the cognitive impairment due to AEDs can lead to adverse consequences. The patients in the age group 12-18 years were selected in this study as a very limited data is available for the cognitive impairment aspect of the AEDs in this age group.

In the present study, the male to female ratio was 70:30 which was higher compared to another study by PiñaGarza JE et al, where the male to female ratio was 53:47 with higher number of male patients (Table 1). ${ }^{9}$

Baseline total MMSE (mean) scores from baseline to 12 weeks changed by $1.30 \pm 1.022$ in the group A (LEV), which were in accordance to a study by Lippa CF et al, at 3 months, the participants who remained on levetiracetam showed excellent cognitive tolerability. ${ }^{10}$

Whereas in group B (VPA), the total MMSE scores deteriorated from the baseline with a mean change of $0.20 \pm 1.40$. The change in both the groups was not statistically significant. Similar results were observed by Fleisher AS et al, in the study (Table 2) and also concluded that valproic acid treatment was associated with accelerated brain volume loss over 1 year using MRI and perhaps with greater cognitive impairment. ${ }^{11}$

Baseline mean total MOCA scores from baseline to 12 weeks changed by $1.17 \pm 1.147$ in the group A (LEV) whereas in group B (VPA), the total MoCA scores deteriorated from the baseline with a mean change of $0.70 \pm 1.119$ (Table 3 ). The change in both the scores from baseline were statistically significant $(p<0.05)$. These results were not in accordance with a study by Gordon $\mathrm{K}$ et al, where valproic acid had a cognitive enhancing effect, probably by reducing epileptiform discharges. ${ }^{12}$ The decrease in the MoCA with the use of valproic acid was similar to a study conducted on the IQ of patients of epilepsy on valproic acid by Khusainova I et al. ${ }^{13}$

Mean baseline total MMSE score in patients with GTCS in group A (LEV) (Table 4) improved significantly by $0.83 \pm 0.753$ from baseline after 12 weeks $(\mathrm{p}<0.05)$. Results were similar to the study by Lippa CF et al, where an improvement in cognition was seen with the use of levetiracetam for 3 months by approximately 2.2 points on MMSE scale in patients with seizures. ${ }^{12}$ Whereas in group B (VPA), the scores deteriorated by $-0.32 \pm 1.314$ (Table 4) similar to the study conducted by Li GH et al, on cognitive impairment. ${ }^{14}$

In patients with partial seizures, mean baseline total MMSE score in group A (LEV) improved significantly by $1.42 \pm 1.060$ from baseline to 12 weeks $(p<0.05)$. Similar results were seen in another study. ${ }^{15}$ Whereas in of patients in group B (VPA) changed by $0.40 \pm 1.817$ similar results were observed in a study by Li GH et al, where an increase in cognition scores were noticed in patients taking valproic acid (Table 4). ${ }^{14}$ Irrespective of the type of epilepsy levetiracetam showed favourable effects of over valproic 
acid on cognitive profile. Mean total MOCA score in patients with GTCS in group A (LEV) improved by from baseline $1.33 \pm 1.366$ to the end of 12 weeks significantly ( $p$ $<0.05)$. Similar findings were reported by $\mathrm{Wu} \mathrm{HL}$ in epileptic patients. ${ }^{16}$ Whereas, in group B (VPA), the total scores deteriorated significantly by $-0.80 \pm 1.080$ similar to the findings in a study by Jiang Y et al, where there was a decrease in the MOCA scores in epileptic patients with the use of valproic acid (Table 4). ${ }^{15}$

In patients of partial seizures, the mean total MOCA scores in patients in group A (LEV) showed significant improvement by $1.13 \pm 1.11$ from the beginning of the study to the end of 12 weeks ( $\mathrm{p}<0.05$ ) (Table 4). Findings were similar to another study in the same regard. ${ }^{16}$ In group B (VPA), there was deterioration in the mean total MoCA scores by $-0.20 \pm 1.304$ (Table 4 ). Similar results were also shown by Zhao J et al. ${ }^{17}$

Various studies have pointed towards the widespread positive effects on cognitive profile in patients of epilepsy with the use of levetiracetam, which may be advantageous specifically in cases associated with the use of pre-existing cognitive deficits.

In this study, cognitive impairment was reported with the use of valproic in many of the MMSE and MoCA parameters that were analyzed. Whereas, improvements in cognition was seen with the levetiracetam on the same parameters.

Seizure frequency at baseline is an important parameter for evaluating the response to treatment in terms of seizure reduction. Seizure frequency at baseline (per month) in group A (LEV) was 1.93 and in group B (VPA) was 2.63 (Table 1). Similar seizure frequencies were seen in a study conducted in Brazil by Li LM et al. ${ }^{18}$

Freedom from seizures (Figure 1) is an important factor for measurement of the efficacy of treatment in epilepsy patients, which was measured by means of a self-reported seizure diary. Mean seizure frequency per month at the beginning of the treatment was $2.63 \pm 1.326$ in VPA group and $1.93 \pm 0.907$ in LEV group (Table 1) similar to the baseline seizure frequency of $1.7 \pm 3.4$ and $2.5 \pm 2.8$ in the VPA and LEV respectively reported by Guilfoyle SM et al. ${ }^{19}$

The patients reporting total seizure freedom at 6 weeks were $83.3 \%$ and $63.3 \%$ in the levetiracetam and valproic acid groups respectively, whereas by $12^{\text {th }}$ week both the groups had achieved complete seizure freedom (Figure 1), which is in accordance with another study by Lowenstein DH et al, where seizure freedom did not vary between newer and older anti-epileptic drugs. ${ }^{20}$

Although, there was a reduction in the seizure frequency in both the groups at 12 weeks from baseline but there was also a significant improvement in cognitive scores with the use of levetiracetam as compared to valproic acid.
Irritability (13\%) and somnolence (7\%) in 4 and 2 patients respectively were the most commonly reported adverse drug reactions in the LEV group followed by tiredness in 1 patient (Table 5). These observed adverse effects were similar to the adverse events observed in another study by Folstein ME et al. ${ }^{7}$

Whereas, in the VPA group headache $(23 \%)$, increased sleep $(17 \%)$, hair fall $(10 \%)$ irritability $(7 \%)$ and weight gain $(7 \%)$ were the most commonly reported adverse effects (Table 5).

As treatment with valproic acid has been associated with a tendency to cause a cognitive decline, regular monitoring for any signs of cognitive impairment is warranted especially in the adolescent and young adult age group where even a slight decline can prove to be harmful.

The data analysing the impact of AEDs on various subdomains of the MMSE and MoCA tests remains limited, due to which the impact of drugs on specific parameters remain ambiguous, therefore the subgroup analysis of various sub-domains of the MoCA and MMSE tests have been done for both the study drugs in this study.

\section{CONCLUSION}

This observational analytical study was done to compare the effect of levetiracetam and valproic acid as monotherapy on cognition in newly diagnosed patients of epilepsy on MMSE and MoCA cognitive assessment tests for a period of 12 weeks.

LEV had a better cognitive profile compared to VPA in all the parameters of MOCA and MMSE. Patients on LEV showed significant improvement compared to VPA in terms of cognitive function. Safety profile of LEV was also better compared to VPA. Irritability and headache were the most common adverse effects in the LEV VPA groups respectively.

LEV, a member of newer anti-epileptic drugs was found to be comparable in efficacy to VPA but with a lesser frequency of adverse effects and had a positive influence on cognition when used as a monotherapy in both the generalized tonic-clonic seizures and partial seizures and maybe considered as an alternative drug to other older AEDs in the treatment of epilepsy.

\section{ACKNOWLEDGEMENTS}

Authors would like to thank Himalayan Institute of Medical Sciences, all patients and colleagues, for their support during study.

Funding: No funding sources
Conflict of interest: None declared
Ethical approval: The study was approved by the
Institutional Ethics Committee (approval no.
SRHU/HIMS/ETHICS/2018/68)




\section{REFERENCES}

1. World Health Organization. Epilepsy. Available at: http://www.who.int/news-room/fact-

sheets/detail/epilepsy/. Accessed 12 July 2018.

2. Vining EP. Cognitive dysfunction associated with antiepileptic drug therapy. Epilepsia. 1987;28:S18-22.

3. Kerr C, Nixon A, Angalakuditi M. The impact of epilepsy on children and adult patient's lives: development of a conceptual model from qualitative literature. Seizure. 2011;20(10):764-74.

4. Holmes GL. Epilepsy in the developing brain: lessons from the laboratory and clinic. Epilepsia. 1997;38(1):12-30.

5. Bourgeois BF. Determining the effects of antiepileptic drugs on cognitive function in pediatric patients with epilepsy. J Child Neurol. 2004;19(1S):S15-24.

6. Hills MD. The psychological and social impact of epilepsy. Neurol Asia. 2007;12(1):10-2.

7. Folstein ME. A practical method for grading the cognitive state of patients for the children. J Psychiatr Res. 1975;12:189-98.

8. Sulheim D, Fagermoen E, Sivertsen $\varnothing \mathrm{S}$, Winger A, Wyller VB, Øie MG. Cognitive dysfunction in adolescents with chronic fatigue: a cross-sectional study. Arch Dis Childhood. 2015;100(9):838-44.

9. Piña-Garza JE, Lagae L, Villanueva V, Renfroe JB, Laurenza A, Williams B, et al. Long-term effects of adjunctive perampanel on cognition in adolescents with partial seizures. Epilepsy Behavior. 2018;83:508.

10. Lippa CF, Rosso A, Hepler M, Jenssen S, Pillai J, Irwin D. Levetiracetam: a practical option for seizure management in elderly patients with cognitive impairment. Am J Alzheimer's Dis Other Dementias®. 2010;25(2):149-54.

11. Fleisher AS, Truran D, Mai JT, Langbaum JB, Aisen PS, Cummings JL, et al. Chronic divalproex sodium use and brain atrophy in Alzheimer disease. Neurol. 2011;77(13):1263-71.

12. Gordon K, Bawden H, Camfield P, Mann S, Orlik P. Valproic acid treatment of learning disorder and severely epileptiform EEG without clinical seizures. J Child Neurol. 1996;11(1):41-3.

13. Khusainova I, Khusainov T. The effect of antiepileptic drugs on IQ in children with epilepsy. J Neurol Sci. 2013;333:e3.

14. Li GH, Shi RF, Wang R, Pang GX, Li JY, Li QH. Influence of lamotrigine and valproate on cognitive function in children with epilepsy. J Applied Clin Pediatrics. 2007;22(12):903.

15. Jiang Y, Hu Y, Wang Y, Zhou N, Zhu L, Wang K. Empathy and emotion recognition in patients with idiopathic generalized epilepsy. Epilepsy Behavior. 2014;37:139-44.

16. Wu HL, Zhao YQ, Ding CY. Analysis of cognitive function impairment and its influencing factors in patients with epilepsy. J Clin Neurol. 2008;(3):19.

17. Zhao J. The Clinical Study about Levetiracetam of Cognitive Function and Emotional Influence in the Patients with Epilepsy-Progress in Modern Biomedicine. Available at: http://en.cnki.com.cn/Article_en/CJFDTotalSWCX201232020.htm Accessed March 152018.

18. Li LM, Fernandes PT, Noronha AL, Marques LH, Borges MA, Borges K, et al. Demonstration project on epilepsy in Brazil: outcome assessment. Arq Neuropsiquiatria. 2007;65:58-62.

19. Guilfoyle SM, Follansbee-Junger K, Smith AW, Combs A, Ollier S, Hater B, et al. Antiepileptic drug behavioral side effects and baseline hyperactivity in children and adolescents with new onset epilepsy. Epilepsia. 2018;59(1):146-54.

20. Lowenstein DH. Seizures and epilepsy. In: Kasper DL, eds. Harrison's Principle of Internal Medicine; $19^{\text {th }}$ ed. New York; McGraw Hill: 2015;2548-2550.

Cite this article as: Khanna $\mathrm{S}$, Bala S, Singh Y, Sharma T, Kalra J, Dhasmana DC, et al. Comparative evaluation of levetiracetam and valproic acid as monotherapy on cognitive impairment in patients of epilepsy. Int $\mathrm{J}$ Basic Clin Pharmacol 2019;8:674-80. 